\title{
Ketamine interactions with gut-microbiota in rats: relevance to its antidepressant and anti-inflammatory properties
}

Bruk Getachew ${ }^{1}$, Joseph I. Aubee ${ }^{2}$, Richard S. Schottenfeld ${ }^{3}$, Antonei B. Csoka ${ }^{4}$, Karl M. Thompson ${ }^{2}$ and Yousef Tizabi ${ }^{*}$

\begin{abstract}
Background: Appreciable evidence suggest that dysbiosis in microbiota, reflected in gut microbial imbalance plays a key role in the pathogenesis of neuropsychiatric disorders including depression and inflammatory diseases. Recently, the antidepressant properties of ketamine have gained prominence due to its fast and long lasting effects. Additional uses for ketamine in inflammatory disorders such as irritable bowel syndrome have been suggested. However, ketamine's exact mechanism of action and potential effects on microbiome is not known. Here, we examined the effects of low dose ketamine, known to induce antidepressant effects, on stool microbiome profile in adult male Wistar rats. Animals (5/group) were injected intraperitoneally with ketamine $(2.5 \mathrm{mg} / \mathrm{kg})$ or saline, daily for 7 days and sacrificed on day 8 when intestinal stools were collected and stored at $-80^{\circ} \mathrm{C}$. DNA was extracted from the samples and the $16 \mathrm{~S}$ rRNA gene-based microbiota analysis was performed using $16 \mathrm{~S}$ Metagenomics application.

Results: At genus-level, ketamine strikingly amplified Lactobacillus, Turicibacter and Sarcina by 3.3, 26 and 42 fold, respectively. Conversely, opportunistic pathogens Mucispirillum and Ruminococcus were reduced by approximately 2.6 and 26 fold, respectively, in ketamine group. Low levels of Lactobacillus and Turicibacter are associated with various disorders including depression and administration of certain species of Lactobacillus ameliorates depressive-like behavior in animal models. Hence, some of the antidepressant effects of ketamine might be mediated through its interaction with these gut bacteria. Additionally, high level of Ruminococcus is positively associated with the severity of irritable bowel syndrome (IBS), and some species of Mucispirillum have been associated with intestinal inflammation. Indirect evidence of anti-inflammatory role of Sarcina has been documented. Hence, some of the anti-inflammatory effects of ketamine and its usefulness in specific inflammatory diseases including IBS may be mediated through its interaction with these latter bacteria.
\end{abstract}

Conclusion: Our data suggest that at least some of the antidepressant and anti-inflammatory effects of daily ketamine treatment for 7 days may be mediated via its interaction with specific gut bacteria. These findings further validate the usefulness of microbiome as a target for therapeutic intervention and call for more detailed investigation of microbiome interaction with central mediators of mood and/or inflammatory disorders.

Keywords: NMDA receptor, Lactobacillus, Turicibacter, Ruminococcus, Sarcina, Mucispirillum, 165 rRNA gene, Metagenomics, Dysbiosis, Inflammatory diseases, Depression

\footnotetext{
* Correspondence: ytizabi@howard.edu

${ }^{1}$ Department of Pharmacology, Howard University College Medicine, 520 W

Street NW, Washington, DC 20059, USA

Full list of author information is available at the end of the article
}

(c) The Author(s). 2018 Open Access This article is distributed under the terms of the Creative Commons Attribution 4.0 International License (http://creativecommons.org/licenses/by/4.0/), which permits unrestricted use, distribution, and reproduction in any medium, provided you give appropriate credit to the original author(s) and the source, provide a link to the Creative Commons license, and indicate if changes were made. The Creative Commons Public Domain Dedication waiver (http://creativecommons.org/publicdomain/zero/1.0/) applies to the data made available in this article, unless otherwise stated. 


\section{Background}

Converging evidence suggests that the brain and the gut microbiota are in bidirectional communication with each other and also with inflammatory processes [1-5]. Thus, on the one hand, dysbiosis, an imbalance in the microbiota community, may occur in depression and chronic stress due to altered brain signaling to the gut [6]. On the other hand, altered gut microbial signaling to the brain may result in brain alterations [7]. Clinical evidence supporting gut microbiota-brain-inflammatory processes interaction, specifically in relation to mood dysregulation such as in major depressive disorder (MDD) include: 1. depressive symptoms are often co-morbid with gastrointestinal (GI) disorders such as metabolic syndrome, inflammatory bowel disease, and irritable bowel syndrome (IBS) [8-12]; 2. this comorbidity presents increased risk for disease progression and poorer outcome, and treatment of one condition can reverse the risk for the other [13-17]; 3. some classes of antibiotics have been shown to have antidepressant effects [18-22]. Conversely, some antidepressants may also possess antimicrobial properties [23, 24]. Moreover, germ-free animals show increased depressive-like behavior that can be reversed by administering a single bacterium such as Bifidobacterium infantis $[25,26]$. Similarly, significant depletion of the gut microbiota with selective antibiotics can result in depressive-like phenotype [27]. Fecal microbiota transplantation from depressed patients to microbiota-depleted rats induces behavioral and physiological characteristic of depression (i.e., anhedonia and altered tryptophan metabolism) in these rats $[4,28]$. Conversely, probiotics consumption can increase plasma levels of tryptophan and reduce levels of the pro-inflammatory cytokines such as interleukin-1-beta (IL-1 $\beta$ ), interleukin-6 (IL-6) and tumor necrosis factor-alpha (TNF $\alpha)$. Interestingly, reduction of these pro-inflammatory cytokines can result in abatement of depressive-like behavior [29]. Overall, these data suggest involvement of microbiome in pathogenesis of depressive behavior and possibly the effectiveness of antidepressants.

Off-label use of ketamine for depression is becoming prominent due to its prompt and sustained antidepressant effects. Indeed, (S)-ketamine (esketamine) is in approval process by FDA as a fast-acting antidepressant with particular application in treatment-resistant depression and suicidal ideation [30]. The purported effects of acute ketamine include inhibition of NMDA (N-methyl-$\mathrm{d}$-aspartate) receptors and activation of AMPA ( $\alpha$-amino-3-hydroxy-5-methyl-4-isoxazolepropionic acid) receptors as well as molecular signaling of mTOR (the mammalian target of rapamycin), which result in enhancement of hippocampal brain-derived neurotrophic factor (BDNF) and increased synaptogenesis [31-35]. The sustained effectiveness of acute ketamine is likely mediated by additional mechanisms as increased BDNF levels are not maintained beyond $24 \mathrm{~h}$ after ketamine administration [36]. In this regard, interaction of acute ketamine with gut microbiota has recently been reported $[37,38]$. Moreover, it was demonstrated that gut microbiome is capable of modulating central BDNF [39, 40]. However, to our knowledge no study has examined the effects of chronic ketamine on gut microbiota.

In addition to its well established antidepressant effects, ketamine has also been advocated for use in inflammatory diseases such as ulcerative colitis [41, 42]. However, here also, no studies on interaction between ketamine and gut microbiota implicated in inflammatory diseases has been carried out. Thus, this study was conducted to determine the effects of chronic ketamine on gut microbiota, especially those implicated in mood regulation and/or inflammatory responses. Our hypothesis was that ketamine would promote microbiota implicated in mood elevation and suppress microbiota implicated in inflammatory diseases.

\section{Methods \\ Animals}

Age matched, approximately 3 months old adult male Wistar rats (Evigo, USA) were housed 2-3 per cage in standard polypropylene shoebox cages $(42 \times 20.5 \times$ $20 \mathrm{~cm}$ ) on hardwood chip bedding (alpha-dry) in a designated room. Throughout the experiment, animals had access to food (Harlan Tek Lab) and water ad libitum. The room was maintained at $24-26^{\circ} \mathrm{C}$ at $51-$ $66 \%$ relative humidity, on a 12 -h light/dark cycle (lights on at $7 \mathrm{am}$ ).

In order to acclimate the subjects to the housing conditions, animals arrived at least one week prior to initiation of any experiment. During this period, they were gentled once daily in order to minimize any stress effects that might result from routine handling.

\section{Drugs}

A ready-made preparation KETAHESIA ${ }^{\bullet}$ inj. Sol. purchased from Henry Schein (Dublin, OH) $(100 \mathrm{mg} / \mathrm{ml}$ ), was diluted with saline to obtain desired concentration of $2.5 \mathrm{mg} / \mathrm{ml}$.

\section{Experimental design}

Following one week of acclimation, the animals were randomly divided into two groups, control and experimental ( $n=5$ each) and were housed in separate cages. Animals belonging to the same group were also randomly selected and housed together (2-3 animals/cage). This housing method assured that the animals in both groups were exposed to identical environment and that there would not be any cross-contaminatin between the treated vs the control group. The number of animals used in each group was based on behavioral observations seen using similar number of animals. 
Control group was injected daily (around noon) with saline, whereas the experimental group received ketamine $(2.5 \mathrm{mg} / \mathrm{kg})$. All injections were done intraperitoneally (i.p.) and were carried out for 7 consecutive days. The volume of injection was $1 \mathrm{ml} / \mathrm{kg}$. The low dose of ketamine used in this study was based on our previous study, where clearly an antidepressant effect of such a dose and duration was observed [32, 43]

\section{Sample collection}

On day 8 , approximately $24 \mathrm{~h}$ after the last ketamine or saline injection, the animals were sacrificed by decapitation, alternating between the groups as described previously [44]. Colons containing stools were collected, quick-frozen on dry ice and stored at $-80^{\circ} \mathrm{C}$. This method of rapid-freezing is considered best-practice for preserving stool DNA samples [45].

\section{Stool DNA extraction}

Total DNA was isolated from stool samples using Norgen's Stool DNA Isolation Kit and the Precellys Dual-24 Homogenizer (Bertin Technologies). Purification was based on spin column chromatography using resin as the separation matrix. Briefly, $200 \mathrm{mg}$ stool samples were bead-homogenized after adding $1 \mathrm{~mL}$ of Lysis Buffer $\mathrm{L}$. One hundred $\mu \mathrm{L}$ of lysis additive was added and vortexed, followed by centrifugation at 20,000 $\mathrm{x} g$ for $5 \mathrm{~min}$. The clear supernatant was transferred $(600 \mu \mathrm{L})$ to a DNAase-free microcetrifuge tube. Next, the samples were centrifuged and $100 \mu \mathrm{L}$ of Binding Buffer I was added to the clean supernatant and incubated on ice for $10 \mathrm{~min}$. Equal amounts of $70 \%$ ethanol were then added to the clean supernatant from Binding Buffer I lysate after centrifugation. The protocol was then followed for complete DNA isolation. The purified DNA was quantified and analyzed for purity using the NanoDrop ${ }^{\mathrm{Tm}} 2000$ Spectrophotometer (NanoDrop Technologies, Wilmington, DE). Twenty $\mu \mathrm{L}$ of purified DNA was then quick-frozen on dry ice and shipped to Norgen Biotek (Thorold, ON, Canada) for 16S rRNA gene analysis.

\section{S rRNA gene sequencing and analysis}

Briefly, the V3-V4 hypervariable region of the bacterial 16S rRNA gene was amplified from $12.5 \mathrm{ng}$ of stool DNA. The amplicons were then cleaned, sequenced according to the Illumina MiSeq 16S Metagenomic Sequencing Library Preparation protocol [46]. The final library was paired-end sequenced at $2 \times 300$ bp using a MiSeq Reagent Kit v3 on the Illumina MiSeq platform. For bioinformatic analysis, the sequencing data was analyzed using the Illumina $16 \mathrm{~S}$ metagenomics app (Illumina 16S Metagenimics Pippeline (v1.0.1) [47], which performs taxonomic classification of 16S rRNA targeted amplicons using an Illumina-curated version of the GeenGenes taxonomic database. The app provides interactive visualization and raw classification output for per-sample and aggregate analyses. Classification was performed using the Illumina $16 \mathrm{~S}$ Metagenomics workflow, which is also available in the MiSeq Reporter software. The algorithm uses a high-performance implementation of the Ribosomal Database Project (RDP) Classifier described in Wang et al., 2007 [48].

\section{Statistical analysis}

Since comparison was performed between two groups with equal variance (ketamine- and saline-treated animals), Student T-test was applied for detecting significant differences in specific measured parameters. The cut-off for statistical significance was $p<0.05$, two-tailed

\section{Results}

\section{Diversity and richness}

Figure 1 depicts the effects of chronic low dose ketamine on gut bacterial diversity (A) and richness (B). A total of 1121 different bacterial species were identified in both saline and ketamine groups. Overall, there was no significant difference in either diversity (Fig. 1a) as estimated by the Shannon Diversity Index (SDI) (saline control vs. ketamine; 2.62 vs. $2.60, P=0.99$ ) or species richness (Fig. 1b) as measured by mean species number. Although a total of 1121 species were identified, only less than 700 (saline control vs. ketamine; 630 vs. $670, P=$ 0.22 ) were considered qualified (i.e., made the cut off at $0.01 \%$ abundance).

\section{Taxa-level distribution}

There were a total number of 29 Phyla, 56 classes, 106 orders, 234 families, 600 genera and 1121 species identified in the two groups. There were no differences between the saline and ketamine group in percent reads, i.e., percentage of identified sequences belonging to each taxon (Fig. 2).

\section{Phylum-level effects}

Ketamine significantly reduced abundances of two phyla, Deferibacteres and Tenericutes (Fig. 3). Overall there were 29 different phyla identified in the two groups. Deferibacteres and Tenericutes are low-abundance phyla accounting for less than $2 \%$ of the total phyla reads. Ketamine selectively reduced Deferibacteres and Tenericutes by approximately 22 and 2 fold, respectively, compared to saline control group.

\section{Class-Level Effects}

Ketamine significantly reduced the levels of two classes, Deferribacteres and Mollicutes by 22 and 2 fold, respectively, compared to the saline group (Fig. 4). There were a total of 56 different classes identified between the two groups. 


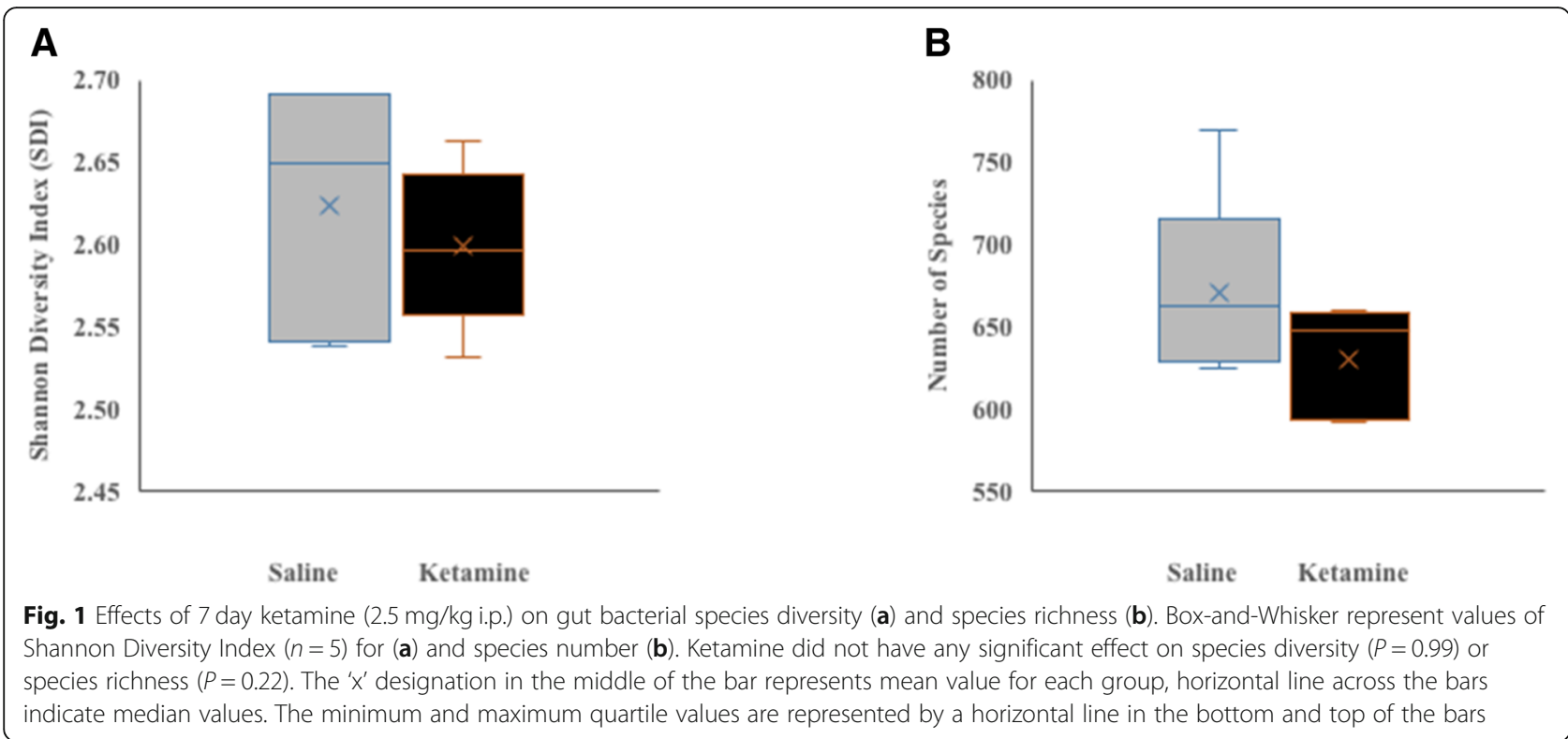

\section{Order-level effects}

Ketamine significantly increased the abundance of Turicibacterales order by 28 fold, and reduced the abundance of four orders: Desulfuromonadales, Deferribacterales, Theromonaerobacterales and Anaeroplasmatales by 2.2, 20, 2 and 3.4 fold, respectively, compared to saline control group (Fig. 5). There were a total of 106 different classes identified in both groups.

\section{Family-level effects}

Ketamine significantly enriched Tuberibacteraceae by 98 fold, Clostridiaceae by 89 fold and Lactobacillaceae by 1.5 fold, whereas Deferrribacteraceae and Ruminococcaceae were reduced by approximately 26 and 2.3 fold, respectively, compared to the saline control group at the family-level (Fig. 6). There were a total of 234 different families. It should be noted that for analysis at the family level, the samples were pooled and hence overall there were two gorups to be compared (control vs treated). Since a statistical analysis could not be perforemed in such cases, we used a conservative cutoff point of a minimum of 1.5 fold difference between the gorups, which could imply important changes.

\section{Genus-level effects}

Ketamine significantly enriched abundances of genera Sarcina, Turicibater, Lactobacillus by 42, 20 and 2 fold respectively, Whereas, levels of Mucispirillum and Ruminococcaceae were decreased by 26.3 and 2.3 fold, respectively, compared to saline control group (Fig. 7).

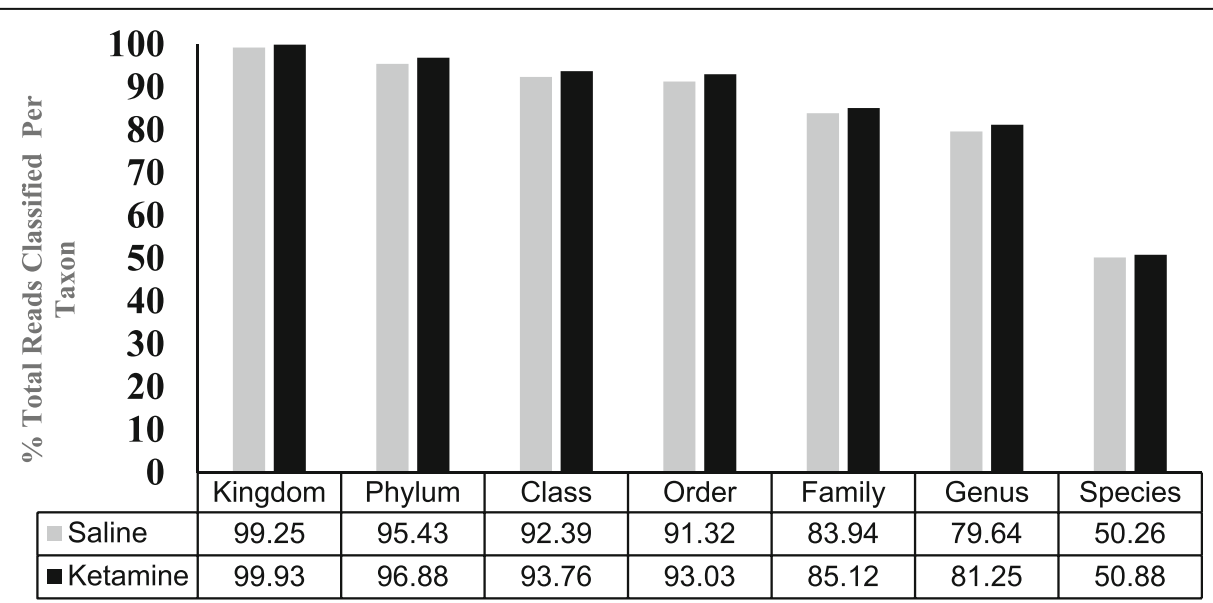

Fig. 2 Effects of 7 day ketamine (2.5 mg/kg i.p.) on gut bacterial distribution of taxa. Values are percent total reads/taxa/group $(n=5)$. There were no significant differences in the \% reads in taxon between saline and ketamine group. Note: the samples from each group were pooled and hence overall there were two groups to be compared (control vs treated) 


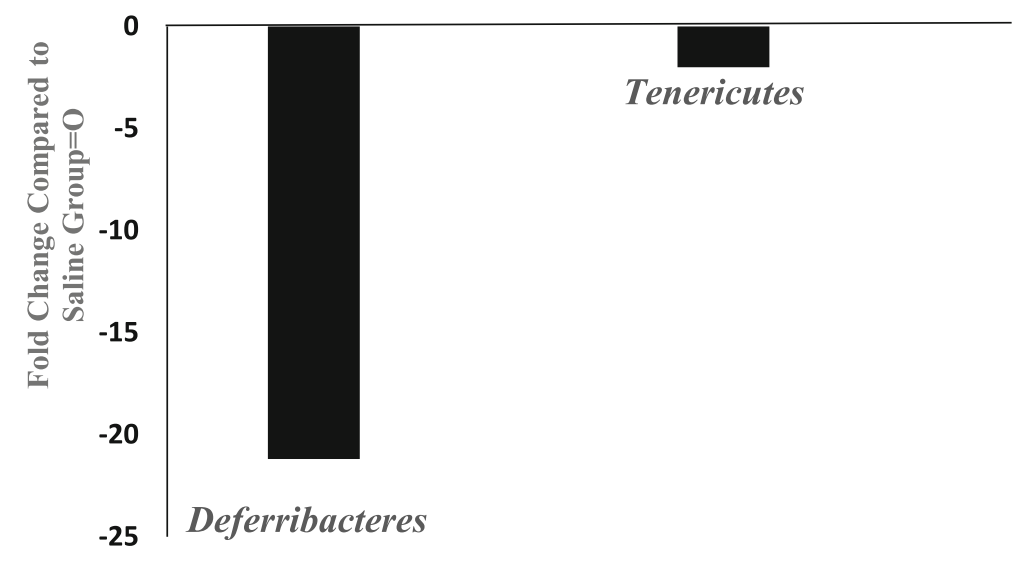

Fig. 3 Effects of 7 day ketamine ( $2.5 \mathrm{mg} / \mathrm{kg}$ i.p.) on fold change in abundance of gut bacterial phylum. Values are the fold change of mean compared to saline-control group $(n=5)$. Ketamine substantially decreased Deferribacteres and Tenericutes compared to saline group by 22 and 2.3 fold, respectively. Note: the samples from each group were pooled and hence overall there were two groups to be compared (control vs treated)

There were a total of 600 different genera. It should be noted that for analysis at the genus level, the samples were pooled and hence overall there were two gorups to be compared (control vs treated). Since a statistical analysis could not be perforemed in such cases, we used a conservative cutoff point of a minimum of 1.5 fold difference between the gorups, which could imply important changes.

\section{Discussion}

Our data indicates drastic effects of chronic low dose ketamine on gut microbial ecology, ranging from 2 to 42 fold changes in specific genera. For example, Lactobacillus and Turicibacter were increased by approximately 2 and 20 fold, respectively, whereas Mucispirillum and Ruminococcus were reduced by approximately 26 and 2.3 fold, respectively. Since low levels of Lactobacillus and Turicibacter are associated with various disorders including depression [49-51], it might be suggested that these gut microbiomes may play a role in ketamine's antidepressant properties. Further support for this contention is provided by findings that administration of Lactobacillus ameliorates depressive-like behavior in animal models [49, 52-54]. As mentioned earlier, antidepressant effects of low dose ketamine have been amply supported in both preclinical as well as clinical studies [30-35].

Conversely, elevated levels of Mucispirillum and Ruminococcus are associated with inflammatory processes [55-59]. Indeed, high levels of Ruminococcus has been shown to be positively associated with the severity of irritable bowel disease and some species of Mucispirillum may cause intestinal inflammation [58-63]. Hence, reduction of these microorganisms by ketamine, may suggest an additional novel mechanism for ketamine's anti-inflammatory effects. On the other hand, since low levels of Sarcina has been implicated in inflammatory processes [64], its 42-fold magnification by ketamine may also be a contributory factor to ketamine's anti-inflammatory properties. Here also, as noted earlier,

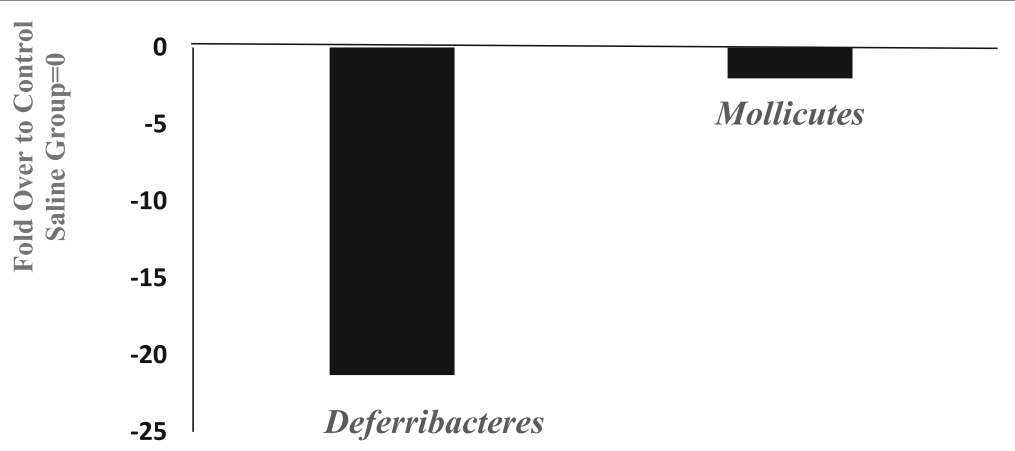

Fig. 4 Effects of 7 day ketamine (2.5 mg/kg i.p.) on fold change in abundance of gut bacterial class. Values are the fold change over saline control group mean $(n=5)$. Ketamine significantly decreased Deferribacteres and Mollicutes compared to saline group by 22 and 2 fold, respectively. Note: the samples from each group were pooled and hence overall there were two groups to be compared (control vs treated) 


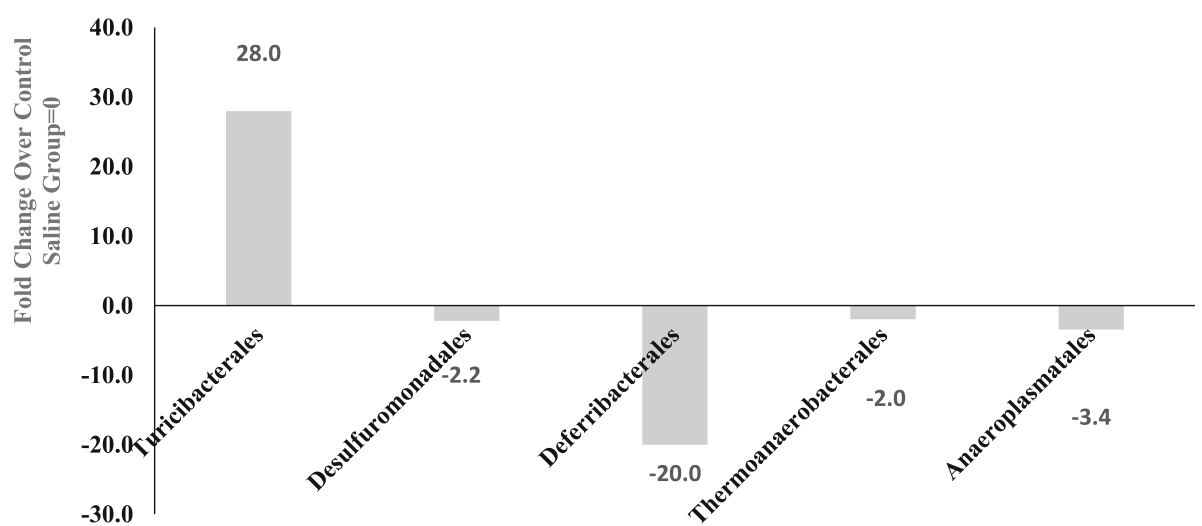

Fig. 5 Effects of 7 day ketamine ( $2.5 \mathrm{mg} / \mathrm{kg}$ i.p.) on fold change in abundance of gut bacterial order. Values are the fold change over saline control group mean $(n=5)$. Ketamine significantly increased Turicibacterales by 28 fold and decreased Desulfuromonadales, Deferribacterales, Theromonaerobacterales and Anaeroplasmatales by 2.2 and 3.4 fold, respectively, compared to saline group. There were a total of 106 different orders. Numbers on the columns refer to the fold-change. Note: the samples from each group were pooled and hence overall there were two groups to be compared (control vs treated)

anti-inflammatory effects of ketamine are well documented [41, 42].

In addition, changes in low-abundance groups such as Lactobaccilus, Sarcina and Turicibacter can markedly influence the gut ecosystem [65, 66]. For example, these groups contain species that can degrade complex polysaccharides to short chain fatty acids such as butyrate $[67,68]$. Changes in these "butyrogenic" bacteria may in turn influence metabolic, inflammatory bowel or neurological/neuropsychiatric disorders [69]. Indeed, butyrate, which can be used as a source of energy by the host, confers many benefits including anti-inflammatory effects [70]. Butyrate can also inhibit histone deacetylase (HDAC), resulting in increased levels of histone acetylation, thereby affecting gene expression [71]. Curiously, modest antidepressant-like effects of sodium butyrate, a weak and highly non-specific inhibitor of class I and class II HDACs, have been reported [72, 73]. Furthermore, by lowering colonic $\mathrm{pH}$, butyrate may confer an added advantage for probiotic microbiota such as Turicibacter, which thrive in lower $\mathrm{pH}$ [68]. Curiously, Westernized diet implicated in many disorders including metabolic syndrome, is linked to lower levels of Lactobacillus and Sarcina, but higher levels of Ruminococcus [64, 74]. Mucispirillum is a gram-negative anaerobic bacterium that can

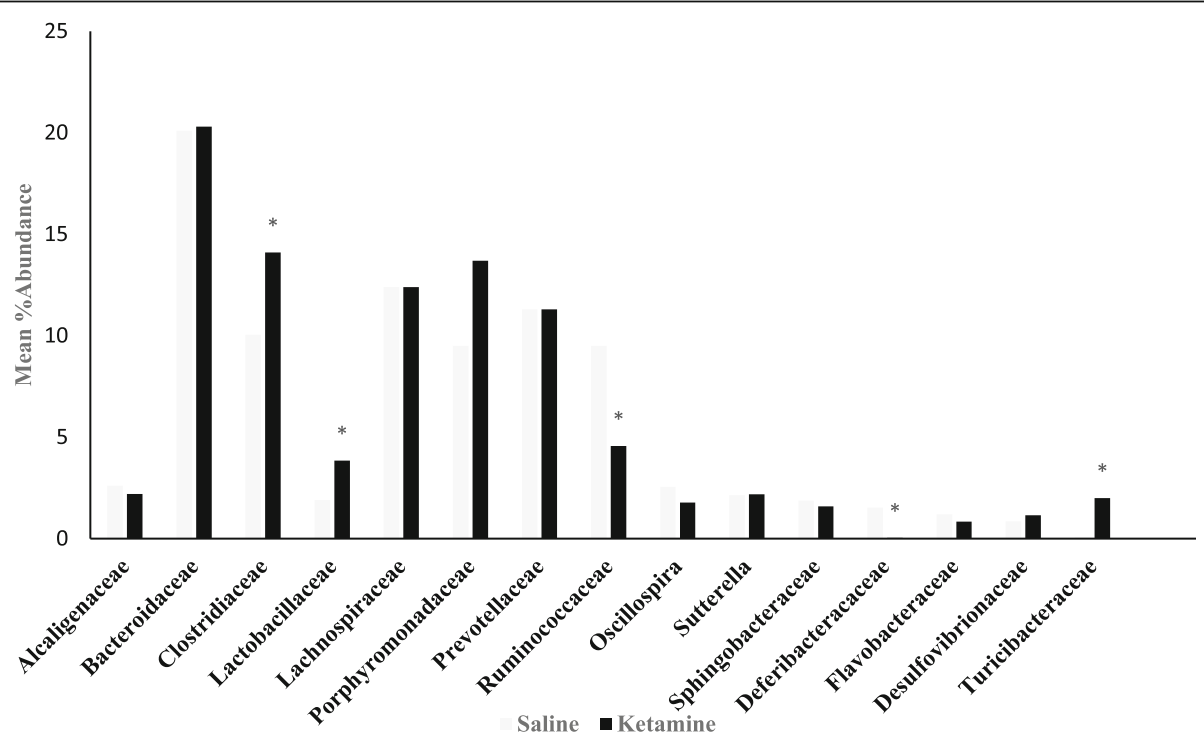

Fig. 6 Effects of 7 day ketamine (2.5 mg/kg i.p.) on percent abundance of gut bacterial family. Values are mean \% abundance of group mean $(n=5)$. Ketamine significantly enriched abundance of genera Lactobacillaceae, Turicibateraceae, Clostridiaceae by $1.5,98$ and 89 fold respectively. Conversely, ketamine reduced abundance of Ruminococcaceae and Deferribacteraceae by 2.3 and 26.3 folds respectively compared to saline group. ${ }^{*}$ indicate significant changes compared to saline. There were a total of 234 families 


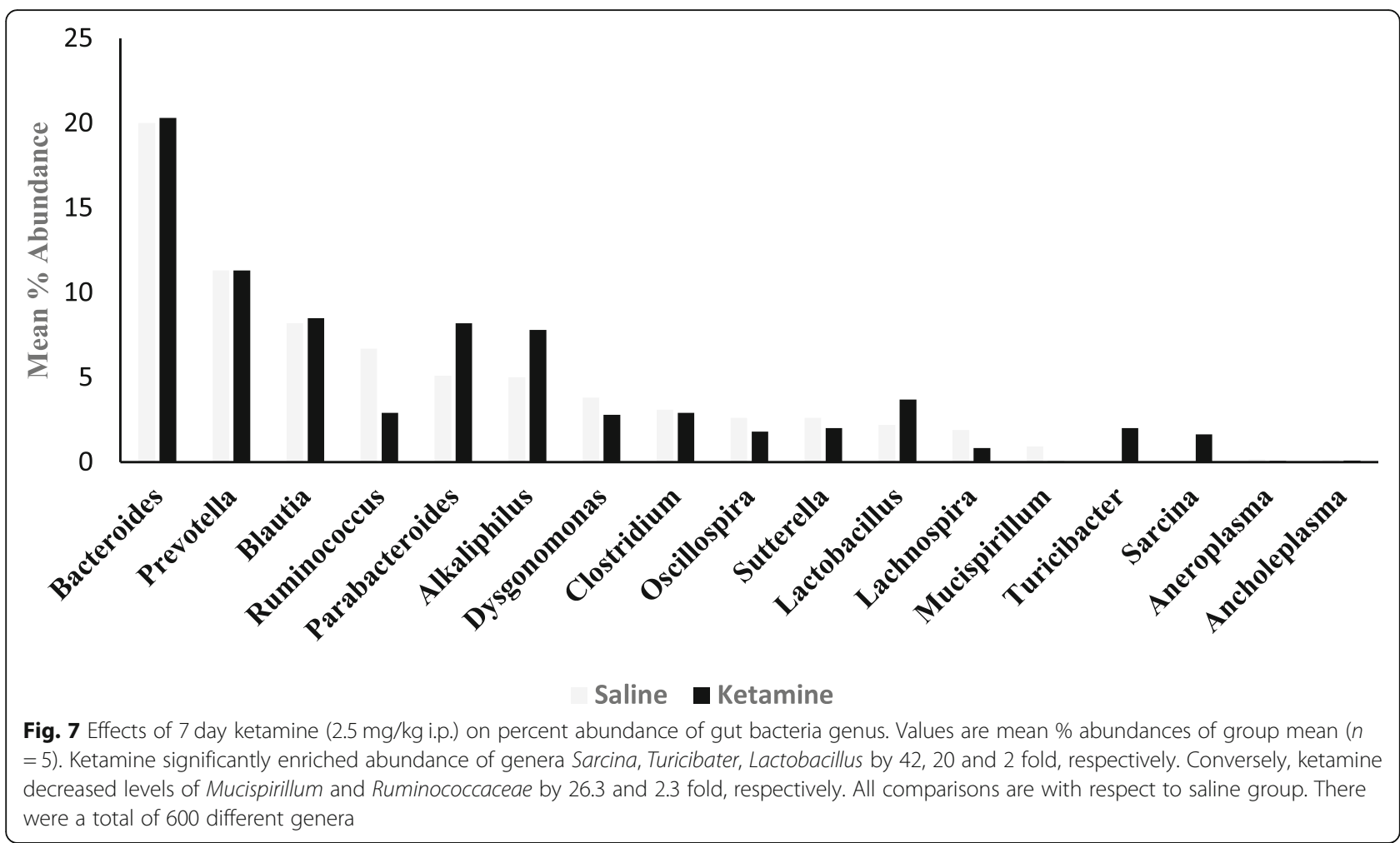

constantly generate lipopolysaccharide (LPS) as an integral component of its outer cell membrane [59, 75]. This and other opportunistic bacteria flourish in gut environment causing inflammation and dysbiosis $[55,57,58]$. To this effect, an association between an increase in Ruminococcus level and diverticulitis, total hip arthroplasty, IBS severity and exercise-induced stress have been reported [56, 59, 76, 78]. Interestingly, exercise-related stress may have similar effects on microbiome. Thus, it was shown that forced exercise reduces levels of Turicibacter, and increases cecal Ruminococcus leading to intestinal inflammation, whereas voluntary wheel running for 6 weeks attenuates symptoms in a colitis mouse model [76].

A recent study by $\mathrm{Qu}$ et al. [37] indicates that acute (R)-ketamine significantly attenuated the increased levels of Ruminococcaceae (a family-taxa of Ruminococcus), in susceptible mice after chronic social defeat. Ketamine's lowering of Ruminococcaceae and reversal of the behavioral deficits, including depressive-like behavior induced by social defeat, suggest that Ruminococcaceae may play a role in stress-induced depressive behavior [37]. Our results also suggest that antidepressant effects of chronic ketamine might be mediated through reduction of Ruminococcus in the gut. On the other hand, ketamine's elevation of gut genera Lactobacillus, Sarcina and Turicibacter reported here, suggests that reestablishment of gut equilibrium by chronic ketamine might be a contributory factor to its anti-inflammatory effects. This contention is further strengthened by findings that ameliorative effects of Turicibacter are correlated with increased intestinal butyric acid [50]. Thus, taken together, it might be suggested that chronic ketamine can ameliorate dysbiotic brought about by diet, stressful exercise or inflammation via its interaction with gut microbiome genera such as Lactobacillus, Sarcina and Turicibacter.

It is also of relevance to note that gut bacteria can influence the colonic mucus layer (mucin), a physical barrier that separates trillions of gut bacteria from the host $[60,62]$. Some microbiota such as Mucispirillum, can increase gut penetrability leading to 'leaky gut' and reduce growth rate of the inner mucus layer [60-62]. Indeed, Mucispirillum, has a potential capacity to degrade mucin by actively destroying the microenvironment of the gut $[60,62]$, leading to leaky gut, which is considered a key contributor to the co-morbid condition of depression and intestinal disorders [77-79]. Moreover, Mucispirillum is positively associated with increases in plasma level of LPS, intestinal inflammation and severity of IBS [59]. Mucispirillum is also considered colitogenic and is used as a microbial marker in active colitis $[58,63]$. Thus, some of the anti-inflammatory and possibly the antidepressant effects of ketamine might also be mediated through reduction of Mucispirillum and gut permeability. In this regard, future characterization of the relationship between Mucispirillum and affect is warranted. 
Elucidation of the direct microbiome influence on neurobiological substrates of mood and on peripheral and central mediators of inflammatory processes may provide novel therapeutic targets in these disorders. It is noteworthy that many animal studies support the notion that central changes in cytokines and BDNF, both of which are directly linked to affective behavior, are influenced by gut microbiota. For example, oral administration of some antimicrobials to mice results in transient alteration of microbiota, increased hippocampal BDNF and antidepressant-like behavior [39]. However, i.p. administration of antimicrobials to mice or oral administration of antimicrobials to germ-free mice do not affect behavior, suggesting that changes in gut microbiomes are necessary to affect central BDNF levels and/or behavior [39]. Thus, inducing changes in the gut microbiota using probiotics, prebiotics or antimicrobial drugs are novel and promising targets in countering affective disorders [40]. In addition, as suggested earlier, manipulation of gut microbiome may also be a novel approach in combatting inflammatory disorders including colitis [80-82]. Therefore, further investigation of the role of specific microbiomes in inflammatory processes and interaction of effective anti-inflammatory compounds with this system can not only enhance our understanding of the gut-brain axis, but can also lead to novel intervention in inflammatory diseases.

\section{Conclusion}

Overall, our findings indicate that chronic administration of ketamine results in significant increases in the levels of low-abundance bacteria genera (e.g. Lactobacillus, Turicibacter and Sarcina), and significant decreases in opportunistic pathogens (e.g. Ruminococcus and Mucispirallum) in male Wistar rats. Thus, it may be suggested that divergent changes in colonic microbiota, where there are increases in probiotic and decreases in pathogenic genera, may in part contribute to the sustained antidepressant and anti-inflammatory effects of ketamine. Clearly, further detailed functional analysis of the role of individual bacterial species and their interactions with central and peripheral mediators of affective behavior or inflammatory processes is warranted. In this vein possible gender effects should also be taken into consideration [83].

\footnotetext{
Abbreviations

AMPA: a-amino-3-hydroxy-5-methyl-4-isoxazolepropionic acid; BDNF: Brain derived neurotrophic factor; CNS: Central nervous system; HDAC: Histone Deacetylase; IBS: Inflammatory bowel syndrome; IL-1 $\beta$ : Interleukin-1-beta; IL6: Interleukin-6; IP: Intraperitoneally; LPS: lipopolysaccharide; MDD: Major depressive disorder; mTOR: Mammalian target of rapamycin; mTOR: The mammalian target of rapamycin; NMDA: N-methyl-d-aspartate; OTU: Operational taxonomic unit; RDP: Ribosomal Database Project; SCFAs: Short chain fatty acids; TNF- a: Tumor necrosis factor
}

\section{Acknowledgments}

Supported by: NIH/NIAAA R03AA022479 (YT); NIH/NIA R21AG047474 (ABC): Howard University Medical Alumni Association (KMT). The authors wish to thank Allison R. Garden for her help in DNA extraction, Dr. Doris E. Hughes and Mr. James $\mathrm{H}$. Bell for their dedicated veterinary services, and Nazar Rghei, Chris O'Brien and Won-Sik Kim from Norgen Biotek for their technical help with 165 rRNA gene sequencing and analysis.

\section{Funding}

Supported by: NIH/NIAAA R03AA022479 (YT); NIH/NIA R21AG047474 (ABC); Howard University Medical Alumni Association (KMT). The funding bodies had no role in the design of the study, collection and analysis of the samples, interpretation of the data or writing of the manuscript.

\section{Availability of data and materials}

The datasets used and/or analyzed during the current study are available from the corresponding author upon request.

\section{Declarations}

All authors declare no conflict of interest.

\section{Authors' contributions}

YT, BG and KMT conceived the study and designed the experiments. ABC, KMT and YT contributed reagents/materials. BG and JIA, performed all experiments. BG, JIA, RSS, ABC, KMT and $Y T$ analyzed data and interpreted results. BG and YT wrote the initial manuscript, which was edited by JIA, RSS, $A B C$ and KMT. All authors have read and have approved the final manuscript.

Ethics and approval and consent to participate

All experiments were carried out in accordance with National Institute of Health $(\mathrm{NIH})$ guidelines and approved by the Howard University Institutional Animal Care and Use Committee (IACUC).

\section{Consent to publication}

Not applicable.

\section{Competing interests}

The authors declare that they have no competing interests.

\section{Publisher's Note}

Springer Nature remains neutral with regard to jurisdictional claims in published maps and institutional affiliations.

\section{Author details}

${ }^{1}$ Department of Pharmacology, Howard University College Medicine, 520 W Street NW, Washington, DC 20059, USA. ²Department of Microbiology, Howard University College Medicine, Washington, DC 20059, USA.

${ }^{3}$ Department of Psychiatry and Behavioral Sciences, Howard University College Medicine, Washington, DC 20059, USA. ${ }^{4}$ Department of Anatomy, Howard University College Medicine, Washington, DC 20059, USA.

Received: 26 March 2018 Accepted: 11 December 2018 Published online: 22 December 2018

\section{References}

1. Raison CL, Capuron L, Miller AH. Cytokines sing the blues: inflammation and the pathogenesis of depression. Trends Immunol. 2006;27(1):24-31.2.

2. Clarke G, Grenham S, Scully P, Fitzgerald P, Moloney RD, Shanahan F, et al. The microbiome-gut-brain axis during early life regulates the hippocampal serotonergic system in a sex-dependent manner. Mol Psychiatry. 2013;18(6): $666-73$

3. Foster JA, Neufeld KAM. Gut-brain axis: how the microbiome influences anxiety and depression. Trends Neurosci. 2013;36(5):305-12.

4. Kelly JR, Borre Y, O'Brien C, Patterson E, El Aidy S, Deane J, et al. Transferring the blues: depression-associated gut microbiota induces neurobehavioural changes in the rat. J Psychiatr Res. 2016;82:109-18. 5

5. Kappelmann N, Lewis G, Dantzer R, Jones PB, Khandaker GM. Antidepressant activity of anti-cytokine treatment: a systematic review and meta-analysis of clinical trials of chronic inflammatory conditions. Mol Psychiatry. 2018;23(2): 335-43. 
6. Clapp M, Aurora N, Herrera L, Bhatia M, Wilen E, Wakefield S. Gut microbiota's effect on mental health: the gut-brain axis. Clin Pract. 2017;7(4):987.

7. Martin CR, Mayer EA. Gut-brain axis and behavior. Nestle Nutr Inst Workshop Ser. 2017:88:45-53.

8. Collins SM, Surette M, Bercik P. The interplay between the intestinal microbiota and the brain. Nat Rev Microbiol. 2012;10(11):735-42.

9. Cryan JF, Dinan TG. Mind-altering microorganisms: the impact of the gut microbiota on brain and behaviour. Nat Rev Neurosci. 2012;13(10):701-12.

10. Bienenstock J, Kunze W, Forsythe P. Microbiota and the gut-brain axis. Nutr Rev. 2015;73(Suppl 1):28-31.

11. De Palma G, Collins SM, Bercik P, Verdu EF. The microbiota-gut-brain axis in gastrointestinal disorders: stressed bugs, stressed brain or both? J Physiol. 2014;592(14):2989-97.

12. Sampson TR, Mazmanian SK. Control of brain development, function, and behavior by the microbiome. Cell Host Microbe. 2015;17(5):565-76.

13. Mikocka-Walus AA. Treatment of psychological co-morbidities in common gastrointestinal and hepatologic disorders. World J Gastrointest Pharmacol Ther. 2010;1(2):64-71.

14. Foxx-Orenstein AE. New and emerging therapies for the treatment of irritable bowel syndrome: an update for gastroenterologists. Therap Adv Gastroenterol. 2016;9(3):354-75

15. Keerthy D, Youk A, Srinath Al, Malas N, Bujoreanu S, Bousvaros A, et al. Effect of psychotherapy on health care utilization in children with inflammatory bowel disease and depression. J Pediatr Gastroenterol Nutr. 2016;63(6):658-64.

16. Sobin WH, Heinrich TW, Drossman DA. Central neuromodulators for treating functional GI disorders: a primer. Am J Gastroenterol. 2017;112(5):693-702.

17. Luo J, Wang T, Liang S, Hu X, Li W, Jin F. Ingestion of lactobacillus strain reduces anxiety and improves cognitive function in the hyperammonemia rat. Sci China Life Sci. 2014;57(3):327-35.

18. Bhalerao S, Talsky A, Hansen K, Kingstone E, Schroeder B, Karim Z, et al. Ciprofloxacin-induced manic episode. Psychosomatics. 2006;47(6):539-40.

19. Volchegorskii IA, Trenina EA. Antidepressant activity of beta-lactam antibiotics and their effects on the severity of serotonin edema. Bull Exp Biol Med. 2006:142(1):73-5.

20. Ahmed AH, Calvird M, Gordon RD, Taylor PJ, Ward G, Pimenta E, et al. Effects of two selective serotonin reuptake inhibitor antidepressants, sertraline and escitalopram, on aldosterone/renin ratio in normotensive depressed male patients. J Clin Endocrinol Metab. 2011:96(4):1039-45.

21. Miyaoka T, Wake R, Furuya M, Liaury K, leda M, Kawakami K, et al. Minocycline as adjunctive therapy for patients with unipolar psychotic depression: an open-label study. Prog Neuro-Psychopharmacol Biol Psychiatry. 2012;37(2):222-6.

22. Mello BS, Monte AS, McIntyre RS, Soczynska JK, Custódio CS, Cordeiro $\mathrm{RC}$, et al. Effects of doxycycline on depressive-like behavior in mice after lipopolysaccharide (LPS) administration. J Psychiatr Res. 2013;47(10): 1521-9.

23. Munoz-Bellido JL, Munoz-Criado S, Garcia-Rodrìguez JA. Antimicrobial activity of psychotropic drugs: selective serotonin reuptake inhibitors. Int J Antimicrob Agents. 2000;14(3):177-80.

24. Lieb J. The immune-stimulating and antimicrobial properties of lithium and antidepressants. J Inf Secur. 2004;49(2):88-93.

25. Desbonnet L, Garrett L, Clarke G, Bienenstock J, Dinan TG. The probiotic Bifidobacteria infantis: an assessment of potential antidepressant properties in the rat. J Psychiatry Res. 2008;43(2):164-74.

26. Campos AC, Rocha NP, Nicoli JR3, Vieira LQ, Teixeira MM, Teixeira AL. Absence of gut microbiota influences lipopolysaccharide-induced behavioral changes in mice. Behav Brain Res. 2016:312:186-94.

27. Hoban AE, Stilling RM, Ryan FJ, Shanahan F, Dinan TG, Claesson MJ, et al. Regulation of prefrontal cortex myelination by the microbiota. Transl Psychiatry. 2016;6:e774

28. Yu M, Jia H, Zhou C, Yang Y, Zhao Y, Yang M, et al. Variations in gut microbiota and fecal metabolic phenotype associated with depression by 165 rRNA gene sequencing and LC/MS-based metabolomics. J Pharm Biomed Anal. 2017:138:231-9.

29. Tan S, Wang Y, Chen K, Long Z, Zou J. Ketamine alleviates depressive-like behaviors via down-regulating inflammatory cytokines induced by chronic restraint stress in mice. Biol Pharm Bull. 2017;40(8):1260-7.

30. Duman RS. Ketamine and rapid-acting antidepressants: a new era in the battle against depression and suicide.F1000Res. 2018;24;7. pii: F1000 Faculty Rev-659. doi: https://doi.org/10.12688/f1000research.14344.1. eCollection 2018. Review.
31. Maeng S, Zarate Jr CA, Du J, Schloesser RJ, McCammon J, Chen G, et al. Cellular mechanisms underlying the antidepressant effects of ketamine: role of a-amino3-hydroxy-5-methylisoxazole-4-propionic acid receptors. 2008;63(4):349-352.

32. Akinfiresoye $L$, Tizabi $Y$. Antidepressant effects of AMPA and ketamine combination: role of hippocampal BDNF, synapsin, and mTOR. Psychopharmacology. 2013;230(2):291-8.

33. Duman RS, Aghajanian GK, Sanacora G, Krystal JH. Synaptic plasticity and depression: new insights from stress and rapid-acting antidepressants. Nat Med. 2016;22(3):238-49.

34. Pałucha-Poniewiera A. The role of glutamatergic modulation in the mechanism of action of ketamine, a prototype rapid-acting antidepressant drug. Pharmacol Rep. 2018;70(5):837-46.

35. Reed JL, Nugent AC, Furey ML, Szczepanik JE, Evans JW, Zarate CA Jr. Ketamine normalizes brain activity during emationally valenced attentional processing in depresssion. Neuroimage Clin. 2018;20:92-101.

36. Garcia LSB, Comim CM, Valvassori SS, Réus GZ, Barbosa LM, Andreazza Stertz $L$, et al. Acute administration of ketamine induces antidepressant-like effects in the forced swimming test and increases BDNF levels in the rat hippocampus. Prog Neuropsychopharmacol Biol Psych. 2008;32(1):140-4.

37. Qu Y, Yang C, Ren Q, Ma M, Dong C, Hashimoto K. Comparison of (R)ketamine and lanicemine on depression-like phenotype and abnormal composition of gut microbiota in a social defeat stress model. Sci Rep. 2017;7(1):15725

38. Yang C, Qu Y, Fujita Y, Ren Q, Ma M, Dong C, Hashimoto K. Possible role of the gut microbiota-brain axis in the antidepressant effects of (R)-ketamine in a social defeat stress model. Transl Psychiatry. 2017;7(12):1294.

39. Bercik P, Collins SM, Verdu EF. Microbes and the gut-brain axis. Neurogastroenterol Motil. 2012;24(5):405-13.

40. Magsood R, Stone TW. the gut-brain axis, BDNF, NMDA and CNS disorders. Neurochemical Research. 2016:41(11):2819-35

41. White M, Shan N, Lindley K, Lloyd-Thomas A, Thomas M. Pain management in fulminating ulcerative colitis. Pediatr Anesth. 2006;16:1148-52.

42. Loix S, Kock M, Henin P. The anti-inflammatory effects of ketamine: state of the art. Acta Anaesthesiol Belg. 2011;62:47-58.

43. Tizabi Y, Bhatti BH, Manaye KF, Das JR, Akinfiresoye L. Antidepressant-like effects of low ketamine dose is associated with increased hippocampal AMPA/NMDA receptor density ratio in female Wistar-Kyoto rats. Neuroscience. 2012;213:2-80

44. Getachew B, Hauser SR, Csoka AB, Taylor RE, Tizabi Y. Role of cortical alpha2 adrenoceptors in alcohol withdrawal-induced depression and tricyclic antidepressants. Drug Alcohol Depend. 2017;175:133-9.

45. Mathay C, Hamot G, Henry E, Georges L, Bellora C, Lebrun L, et al. Method optimization for fecal sample collection and cecal DNA extraction biopreserv biobank 2015;13(2):79-93.

46. Illumina MiSeq 165 metagenomic sequencing library preparation protocol. http://support.illumina.com/downloads/16s_metagenomic_sequencing library_preparation. Accessed 25 Aug 2017.

47. Illumina 165 metagenomics pipeline (v1.0.1). https://basespace.illumina.com/ apps/593593/16S-Metagenomics/perferredversion. Accessed 20 Aug 2017.

48. Wang Q, Garrity GM, Tiedje JM, Cole JR. Naive Bayesian classifier for rapid assignment of rRNA sequences into the new bacterial taxonomy. Appl Environ Microbiol. 2007;73(16):5261-7

49. Bravo JA, Forsythe $P$, Chew MV, Escaravage $E$, Savignac HM, Dinan TG, et al. ingestion of lactobacillus strain regulates emotional behavior and central GABA receptor expression in a mouse via the vagus nerve. PNAS. 2011; 108(38):16050-5.

50. Zhong Y, Nyman M, Fåk F. Modulation of gut microbiota in rats fed high-fat diets by processing whole-grain barley to barley malt. Mol Nutr Food Res. 2015;59(10):2066-76

51. Kunugi H. Depressive disorder and gut-brain interaction. Brain and Nerve. 2016;68(6):641-6.

52. Romijn AR, Rucklidge JJ, Kuijer RG. A double-blind, randomized, placebocontrolled trial of lactobacillus helveticus and Bifidobacterium longum for the symptoms of depression. Aust N Z J Psychiatry. 2017;51(8):810-21.

53. Slykerman RF, Hood F, Wickens K, Thompson JMD, Barthow C, Murphy R, et al. Effect of lactobacillus rhamnosus HNOO1 in pregnancy on postpartum symptoms of depression and anxiety: a randomised double-blind placebocontrolled trial. EBioMedicine. 2017:24:159-65.

54. Ng QX, Peters C, Yih C, Ho X, Yutong D, Limd Yeo WS. A meta-analysis of the use of probiotics to alleviate depressive symptoms. J Affect Disord. 2018;228:13-9. 
55. Gaboriau-Routhiau V, Rakotobe S, Lécuyer E, Mulder I, Lan A, Bridonneau C, et al. The key role of segmented filamentous bacteria in the coordinated maturation of gut helper T cell responses. Immunity. 2009;31(4):677-89.

56. Hansen SKG, Skov MN, Justesen US. Two cases of Ruminococcus gnavus bacteremia associated with diverticulitis. J Clin Microbiology. 2013;51(4):1334-6.

57. Lennon P, Saunders J, Fenton JE. A longer stay for the kissing disease: epidemiology of bacterial tonsillitis and infectious mononucleosis over a 20year period. J Laryngol Otol. 2013;127(2):187-91.

58. El Aidy S, Dinan TG, Cryan JF. Immune modulation of the brain-gut-microbe axis. Front Microbiol. 2014:5:164.

59. Moreno-Indias I, Yorres M, Sanchez-Alcohlado L, Cardona F, Almendros I, Gozal D, et al. Normoxic recovery mimicking treatment of sleep apnea does not reverse intermittent hypoxia-induced bacterial dysbiosis and low-grade endotoxemia in mice. Sleep. 2016;39(10):1891-7.

60. Robertson BR, O'Rourke JL, Neilan BA, Vandamme P, On SL, Fox JG, et al. Mucispirillum schaedleri gen. Nov., sp. nov., a spiral-shaped bacterium colonizing the mucus layer of the gastrointestinal tract of laboratory rodents. Int. J Syst Evolut Microbiol. 2005;55(3):1199-204.

61. Bailey MT, Dowd SE, Galley JD, Hufnagle AR, Allen RG, Lyte M. Exposure to a social stressor alters the structure of the intestinal microbiota: implications for stressor-induced immunomodulation. Brain Behav Immun. 2011;25(3): 397-407.

62. Berry D, Schwab C, Milinovich G, Reichert J. Phylotype-level 165 rRNA analysis reveals new bacterial indicators of health state in acute murine colitis. ISME J. 2012;6(11):2091-106.

63. Rooks MG, Veiga P, Wardwell-Scott LH, Tickle T, Segata N, Michaud M, et al. Gut microbiome composition and function in experimental colitis during active disease and treatment-induced remission. ISME J. 2014;8(7):1403-17.

64. Rossi G, Pengo G, Caldin M, Palumbo Piccionello A, Steiner JM, et al. Comparison of microbiological, histological, and immunomodulatory parameters in response to treatment with either combination therapy with prednisone and metronidazole or probiotic VSL\#3 strains in dogs with idiopathic inflammatory bowel disease. PLoS One. 2014;9(4):e94699.

65. Hausmann B, Knorr KH, Schreck K, Tringe SG, Glavina Del Rio T, Loy AM Consortia of low-abundance bacteria drive sulfate reduction-dependent degradation of fermentation products in peat soil microcosms. ISME J. 2016 ; 10(10):2365-75.

66. Yan W, Sun C, Yuan J, Yang N. Gut metagenomic analysis reveals prominent roles of lactobacillus and cecal microbiota in chicken feed efficiency. Sci Rep. 2017;7:45308.

67. Duncan $\mathrm{SH}$, Louis $\mathrm{P}$, Flint HJ. Lactate-utilizing bacteria, isolated from human feces, that produce butyrate as a major fermentation product. Appl Environ Microbiol. 2004;70(10):5810-7.

68. Biddle AS, Black SJ, Blanchard JL. An in vitro model of the horse gut microbiome enables identification of lactate-utilizing bacteria that differentially respond to starch induction. PLoS One. 2013;8(10):e77599.

69. Stilling RM, van de Wouw M, Clarke G, Stanton C, Dinan TG, Cryan JF. The neuropharmacology of butyrate: the bread and butter of the microbiotagut-brain axis? Neurochem Int. 2016;99:110-32.

70. Donohoe DR, Curry KP, Bultman SJ. Microbial oncotarget: bacterialproduced butyrate, chemoprevention and Warburg effect. Oncotarget. 2013;4(2):182-3.

71. Davie JR. Inhibition of histone deacetylase activity by butyrate. J Nutr. 2003; 133(7):2485S-93S.

72. Tsankova NM, Berton O, Renthal W, Kumar A, Neve RL, Nestler EJ. Sustained hippocampal chromatin regulation in a mouse model of depression and antidepressant action. Nat Neurosci. 2006;9(4):519-25.

73. Schroeder FA, Gilbert TM, Feng N, Taillon BD, Volkow ND, Innis RB, et al. Expression of HDAC2 but not HDAC1 transcript is reduced in dorsolateral prefrontal cortex of patients with schizophrenia. ACS Chem Neurosci. 2017; 8(3):662-8.

74. Martínez I, Stegen JC, Maldonado-Gómez MX, Eren AM, Siba PM, Greenhill AR, et al. The gut microbiota of rural Papua new Guineans: composition, diversity patterns, and ecological processes. Cell Rep. 2015;11(4):527-38.

75. Erridge C, Moncayo-Nieto OL, Morgan R, Young M, Poxton IR. Acinetobacter baumannii lipopolysaccharides are potent stimulators of human monocyte activation via toll-like receptor 4 signalling. J Med Microbiol. 2007:56(2):165-71.

76. Allen JM, Berg Miller ME, Pence BD, Whitlock K, Nehra V, Gaskins HR, et al. Voluntary and forced exercise differentially alters the gut microbiome in c57bl/6j mice. J Appl Physiol. 2015;118(8):1059-66.
77. Dantzer R, O'Connor JC, Freund GG, Johnson RW, Kelley KW. From inflammation to sickness and depression: when the immune system subjugates the brain. Nat Rev Neurosci. 2008;9(1):46-56.

78. Cani PD, Bibiloni R, Knauf C, Waget A, Neyrinck AM, Delzenne NM, et al. Changes in gut microbiota control metabolic endotoxemia-induced inflammation in high-fat diet-induced obesity and diabetes in mice. Diabetes. 2008;57(6):1470-81

79. Turnbaugh PJ, Gordon J. The core gut microbiome, energy balance and obesity. J Physiol. 2009;587(17):4153-8.

80. Laserna-Mendieta EJ, Clooney AG, Carretero-Gomez JF, Moran C, Sheehan D, Nolan JA, et al. Determinants of reduced genetic capacity for butyrate synthesis by the gut microbiome in Crohn's disease and ulcerative colitis. J Crohns Colitis. 2018;12(2):204-16.

81. Goyal A, Yeh A, Bush BR, Firek BA, Siebold LM, Rogers MB, et al. Safety, clinical response, and microbiome findings following fecal microbiota transplant in children with inflammatory bowel disease. Inflamm Bowel Dis. 2018;24(2):410-21.

82. Webb RC, Bakker DH, Koboziev I, Jones-Hall Y, Kottapalli RK, Ostanin D, et al. Differential susceptibility to $T$ cell-induced colitis in mice: role of the intestinal microbiota. Inflamm Bowel Dis. 2018;24(2):361-79.

83. Becker JB, Koob GF. Sex differences in animal models: focus on addiction. Pharmacol Rev. 2016;68:242-63.

\section{Ready to submit your research? Choose BMC and benefit from:}

- fast, convenient online submission

- thorough peer review by experienced researchers in your field

- rapid publication on acceptance

- support for research data, including large and complex data types

- gold Open Access which fosters wider collaboration and increased citations

- maximum visibility for your research: over $100 \mathrm{M}$ website views per year

At BMC, research is always in progress.

Learn more biomedcentral.com/submissions 